\title{
HAPPi Kneecaps! A double-blind, randomised, parallel group superiority trial investigating the effects of sHoe inserts for adolescents with patellofemoral Paln: phase II feasibility study
}

\author{
Isobel C. O'Sullivan', Kay M. Crossley², Steven J. Kamper ${ }^{3,4}$, Marienke van Middelkoop ${ }^{5}$, Bill Vicenzino',
} Melinda M. Franettovich Smith ${ }^{1}$, Hylton B. Menz ${ }^{6}$, Anne J. Smith ${ }^{7}$ Kylie Tucker ${ }^{8}$, Karina T. O'Leary ${ }^{9,10}$,

Nathalia Costa ${ }^{1}$ and Natalie J. Collins ${ }^{1,2^{*}}$ (D)

\begin{abstract}
Background: Patellofemoral pain (PFP) affects one-third of adolescents and can persist into adulthood, negatively impacting health and quality of life. Foot orthoses are a recommended treatment for adults with PFP, but have not been evaluated in adolescents. The primary objective was to determine the feasibility of conducting a full-scale randomised controlled trial $(\mathrm{RCT})$ evaluating effects of contoured, prefabricated foot orthoses on knee pain severity and patient-perceived global change, compared to flat insoles. The secondary objective was to describe outcomes on a range of patient-reported outcome measures.

Methods: We recruited adolescents aged 12-18 years with PFP of $\geq 2$ months duration into a double-blind, randomised, parallel-group feasibility trial. Participants were randomised to receive prefabricated contoured foot orthoses or flat shoe insoles, and followed for 3 months. Participants and outcome assessors were blinded to group allocation. Primary outcomes were feasibility of a full-scale RCT (number of eligible/enrolled volunteers; recruitment rate; adherence with the intervention and logbook completion; adverse effects; success of blinding; drop-out rate), and credibility and expectancy of interventions. Secondary outcomes were patient-reported measures of pain, symptoms, function, quality of life, global rating of change, patient acceptable symptom state, and use of cointerventions.
\end{abstract}

\footnotetext{
* Correspondence: n.collins1@uq.edu.au

'School of Health and Rehabilitation Sciences: Physiotherapy, The University of Queensland, Brisbane, Australia

${ }^{2}$ La Trobe Sport and Exercise Medicine Research Centre, School of Allied Health, Human Services and Sport, La Trobe University, Melbourne, Australia Full list of author information is available at the end of the article
}

C C The Author(s). 2021 Open Access This article is licensed under a Creative Commons Attribution 4.0 International License, which permits use, sharing, adaptation, distribution and reproduction in any medium or format, as long as you give appropriate credit to the original author(s) and the source, provide a link to the Creative Commons licence, and indicate if changes were made. The images or other third party material in this article are included in the article's Creative Commons licence, unless indicated otherwise in a credit line to the material. If material is not included in the article's Creative Commons licence and your intended use is not permitted by statutory regulation or exceeds the permitted use, you will need to obtain permission directly from the copyright holder. To view a copy of this licence, visit http://creativecommons.org/licenses/by/4.0/ The Creative Commons Public Domain Dedication waiver (http://creativecommons.org/publicdomain/zero/1.0/) applies to the data made available in this article, unless otherwise stated in a credit line to the data. 
Results: 36 out of 279 (12.9\%) volunteers (27 female, mean (SD) age 15 (2) years, body mass 60 (13) kg) were eligible and enrolled, at a recruitment rate of 1.2 participants/week. 17 participants were randomised to receive foot orthoses, and 19 to flat insoles. 15 participants returned logbooks; 7/15 (47\%) adhered to the intervention. No serious adverse events were reported. 28\% (10/36, 4 pandemic-related) of participants dropped out before 3 months. Blinding was successful. Both groups found the inserts to be credible.

Conclusions: Based on a priori criteria for feasibility, findings suggest that a full-scale RCT comparing contoured foot orthoses to flat insoles in adolescents with PFP would not be feasible using the current protocol. Prior to conducting a full-scale RCT, feasibility issues should be addressed, with protocol modifications to facilitate participant retention, logbook completion and shoe insert wear.

Trial registration: Australian New Zealand Clinical Trials Registry (ANZCTR): ACTRN12619000957190. Date registered: 8/07/2019.

Keywords: Patellofemoral pain, Adolescents, Foot orthoses, Physiotherapy, Feasibility

\section{Background}

Patellofemoral pain (PFP) is a common musculoskeletal condition affecting $30 \%$ of adolescents [1]. Pain is often aggravated by activities involving weightbearing on a flexed knee; for example, running, jumping, ascending and descending stairs, and squatting. Pain and disability associated with PFP in adolescents can affect participation in physical activity and sport, general and mental health, and quality of life [2-6]. PFP is persistent, even in adolescents, with $21 \%$ of 12 - to 35 -year-olds still reporting pain 6 years after an initial appointment with a general practitioner [7].

There are few evidence-informed recommendations to treat PFP in adolescents and thus, interventions developed for use in adults tend to be used in adolescents, often with poorer outcomes $[8,9]$. Key differences between adolescents and adults may underpin worse outcomes in adolescents. Specifically, adolescents with greater hip abduction strength have an increased risk of PFP, while there is no association in adults [10]. Adults tend to present with reduced quadriceps strength [10], while adolescents demonstrate no quadriceps deficit until 16-18 years of age [4]. The presence of bilateral symptoms also differs between groups, with $55-60 \%$ of adults reporting bilateral symptoms [11, 12] compared to $70-79 \%$ of adolescents [13]. Poorer adherence to recommended treatments, particularly exercise therapy, also likely contributes to worse outcomes in adolescents [9]. Only two randomised controlled trials (RCTs) have investigated treatments specifically for adolescents with PFP $[13,14]$. Their findings indicate that exercise therapy improves outcomes in this group, with further improvements arising when soft foot orthoses were prescribed [13, 14].

Foot orthoses are inserts worn in shoes that are contoured to the shape of the foot, and were recommended to treat PFP in the 2018 consensus statement [8]. Our previous work demonstrated the effectiveness of foot orthoses in adults with PFP $[11,15]$. Compared to participants allocated to flat insoles or wait and see, adults with PFP who received prefabricated foot orthoses demonstrated greater global improvement at 6 weeks [11, 15], and a faster improvement in symptom severity [11]. This is important because duration and severity of symptoms predict poor long-term prognosis for PFP [16]. Although important preliminary information about the effects of foot orthoses in 10 female adolescents with PFP was provided in a pilot study [14], a modern approach to foot orthoses prescription with a larger, more diverse adolescent population is required. To inform the conduct of a full-scale RCT evaluating foot orthoses for adolescents with PFP, a feasibility trial is needed to determine whether contemporary clinical trial methods and foot orthoses prescription are feasible in this population.

The primary objective of this study was to determine the feasibility of conducting a full-scale RCT evaluating the effects of contoured, prefabricated foot orthoses on knee pain severity and patient-perceived global change in adolescents with PFP, compared to flat insoles. The secondary objective was to describe outcomes on a range of patient-reported outcome measures.

\section{Methods}

\section{Experimental design}

The HAPPi Kneecaps! Study (sHoe inserts for Adolescents with Patellofemoral PaIn) was a randomised, controlled, participant- and assessor-blind feasibility trial with two parallel groups (1:1 allocation ratio). Detailed study methods are available in the protocol paper [17]. We consulted the SPIRIT 2013 statement [18] and the CONSORT 2010 statement extension to randomised pilot and feasibility trials [19] in designing and reporting the study. Guidelines for trials modified due to COVID- 
19 [20] were also consulted. The University of Queensland Human Research Ethics Committee granted ethics approval (approval number 2018000159). The trial was prospectively registered on the Australia New Zealand Clinical Trials Registry on 08/07/2019 (ACTRN12619000957190).

\section{Participants}

Adolescent volunteers were recruited from the community in Brisbane and the Gold Coast, Queensland, Australia. Several methods were used for recruitment, including advertising at community and school sporting events, and on websites and social media pages (Facebook, Instagram). Participants were also recruited from our existing database of PFP volunteers.

The inclusion criteria were: (i) aged $12-18$ years; (ii) non-traumatic onset of anterior pain rated at least 3 on an 11 -point numerical rating scale $(0=$ no pain, $10=$ worst pain imaginable); (iii) pain aggravated by PFJloading activities (e.g., squatting, stair ambulation, running, jumping); and (iv) pain present for two months or more, and at some time during most weeks. Exclusion criteria were: (i) concomitant pain at sites other than the anterior knee (e.g. other knee structures, hip, lumbar spine); (ii) history of surgery on the ipsilateral limb or lumbar spine, or other suspected knee joint pathology (e.g. Osgood Schlatter's Disease); (iii) planned lower limb surgery; (iv) recent PFP treatment (e.g. physiotherapy or knee joint injections in the last 3 months; foot orthoses prescription in the last 12 months); or (v) any foot condition impeding the prescription of foot orthoses.

All participants were required to provide written informed consent prior to participation. For participants under 18 years of age, their parent/guardian was also required to give written informed consent.

\section{Sample size}

Formal sample size calculations were not conducted [21, $22]$. We estimated that 40 participants (20 participants per group) would allow us to observe practicalities of recruitment, acceptability of the shoe inserts, adverse events, dropouts, and sample variability.

\section{Procedure}

Eligibility was determined by one investigator (ICO, registered Physiotherapist) through two phases: (i) online screening (Research Electronic Data Capture [REDCap], Vanderbilt University, Nashville, USA); and (ii) physical screening at The University of Queensland to confirm the clinical diagnosis of PFP. Participants (and their parent/guardian if aged under 18 years) gave written consent and, after baseline measures were obtained, were randomised to receive either contoured foot orthoses or flat shoe inserts. We used simple randomisation, with the randomisation sequence generated by one investigator (KT) using a random number generator. The research assistant (KTO) held the randomisation sequence off-site to ensure concealed allocation, and was responsible for communicating group allocation to the physiotherapists. Study physiotherapists were unable to be blinded to group allocation due to the nature of the intervention. The primary investigator (ICO) was blinded to group allocation throughout the trial and collected primary outcomes. Secondary outcomes were self-reported by participants. Blinding of participants was maintained through communicating to each participant that there were two interventions that were being investigated, however, the two types of interventions and study hypotheses were not disclosed [11, 23].

\section{Interventions}

Participants attended physiotherapy appointments at one of six clinics across Brisbane and the Gold Coast for fitting of their allocated shoe insert. Study physiotherapists were trained in fitting procedures, as used in previous studies to maximise comfort [23]. Each participant received up to four pairs of inserts, fit to shoes that would accommodate the inserts and provide support. Participants were asked to attend up to three appointments to allow for any adjustments to be made and ensure inserts were comfortable. Each person was asked to wear the inserts as much as possible throughout the week. Participants in both groups received a handbook, which provided general information and advice about PFP and activity [17].

\section{Prefabricated contoured foot orthoses}

Participants assigned to the contoured foot orthoses group received prefabricated foot orthoses (Vasyli Medical, Labrador, Australia). The orthoses are manufactured from ethylene-vinyl acetate (EVA) with options for a high (hard, Shore A $70^{\circ}$ ), medium (Shore A 55 ) and low (soft, Shore A $45^{\circ}$ ) density orthosis with inbuilt medial arch support and varus wedging. Density was selected based on comfort, and modifications were made to the orthoses in the form of heat moulding or wedges to achieve a comfortable fit [17], based on our published algorithm [23].

\section{Flat shoe insoles}

Participants assigned to this group received flat shoe insoles made of the same high-density EVA as the contoured foot orthoses, which were a uniform thickness of $3 \mathrm{~mm}$ along their length. To facilitate blinding regarding the true intervention, the contoured foot orthoses and flat insoles were covered in the same fabric and markings. Participants randomised to this group were advised that the intervention enhanced sensory feedback, and 
the insoles were heat moulded as necessary to enhance comfort [17].

\section{Outcome assessment}

At baseline, participant details were recorded, including demographics, knee/s affected, symptom duration and aggravating activities. Patient-reported outcome measures were collected at baseline, 6 weeks, and 3 months post-randomisation via an online platform (REDCap). For the duration of the study, participants were asked to complete a paper logbook to record details of daily activities, pain, and footwear [17]. Study physiotherapists recorded attendance, prescription notes and adverse events during fitting and follow-up appointments. 3 months was nominated as the primary endpoint.

The primary outcome was the feasibility of conducting a full-scale RCT. Feasibility was assessed by evaluating the following outcomes:

(i) Number of eligible volunteers (from recruitment database).

(ii) Willingness of participants to enrol in the study (from recruitment database).

(iii) Recruitment rate (from recruitment database).

(iv) Adherence with allocated intervention and logbook completion (from participant logbook).

(v) Adverse events (from Study Practitioner notes, adverse events database, and participant logbook).

(vi) Success of blinding (risk of performance and detection bias, from the Credibility and Expectancy Questionnaire) [24].

(vii) Drop-out rate (from trial database; defined as participants who did not complete 3-month outcome measures).

Participants completed the Credibility and Expectancy Questionnaire [24] at baseline and at their second visit with the study physiotherapist ( 2 weeks postrandomisation). The Credibility and Expectancy Questionnaire consists of six items in two sections; four items related to thoughts, and two items related to feelings. Credibility was derived from the first three thought items, and expectancy was derived from the fourth thought question and the two feeling questions [25]. The Credibility and Expectancy Questionnaire has been used in previous adolescent studies [26, 27].

Prior to the study, we set three criteria to determine the feasibility of a full-scale RCT: (i) a recruitment rate of 1 participant per week; (ii) adherence of at least $2 \mathrm{~h}$ of shoe insert wear per day for 5 days a week; and (iii) $\leq 20 \%$ drop out rate at 3 months [17].

The following secondary outcomes were collected by online questionnaires and participant logbooks (details provided in the protocol paper) [17]: (i) Usual and worst knee pain severity during a selfnominated aggravating activity, measured using a $100 \mathrm{~mm}$ visual analogue scale $(0 \mathrm{~mm}=$ no pain and $100 \mathrm{~mm}=$ worst pain imaginable) [28].

(ii) Knee injury and Osteoarthritis Outcome Score Child Version (KOOS-Child) [29].

(iii) Knee injury and Osteoarthritis Outcome Score Patellofemoral Subscale (KOOS-PF) [30].

(iv) Global Rating of Change (GROC), using a 7-point Likert scale [13].

(v) Patient Acceptable Symptom State (PASS) [31].

(vi) Anterior Knee Pain Scale [32].

(vii) EuroQOL-5D-5L. The UK time-trade-off scoring algorithm was used to weight each participant's profile data to produce a single EQ-5D index score (as there are no data published in Australian adult or adolescent populations) [33].

(viii)Use of co-interventions (e.g., pain medication, physiotherapy, knee brace, other footwear interventions).

\section{Statistical analyses}

Analyses were conducted by a blinded investigator (ICO) using SPSS software (IBM SPSS Statistics for Macintosh, Version 27.0, IBM Corp., Armonk, NY, USA). Baseline data were checked for normality using Shapiro-Wilk tests and presented as mean and standard deviation (normal distribution) or median and interquartile range (not normal distribution). Primary feasibility outcomes for this study were presented using descriptive statistics. Descriptive statistics were calculated for secondary outcomes at all timepoints, separately for each group.

\section{Protocol deviations}

There are two instances where our methods deviated from those reported in our protocol paper [17]. We were not able to report data from the Youth Quality of Life Short Form at any of the measured timepoints. This was due to an error with the online data collection platform. We also planned to provide a range of plausible estimates of treatment effects for prefabricated foot orthoses, compared to flat insoles, by reporting between-group differences (with 95\% confidence intervals) for secondary outcome measures. Due to experiencing greater loss to follow-up than anticipated (including the impact of COVID-19), and the likely imprecision of between-group estimates from a small pilot trial [34], we elected not to report this. Instead, we only present descriptive statistics for secondary outcomes at each timepoint.

\section{Results}

Between August 2019 and March 2020, 260 volunteers responded to advertisements and completed online 
screening (Fig. 1). An additional 19 volunteers were recruited from a pre-existing database. 149 volunteers were potentially eligible after online screening. Of these, 97 did not respond to email or telephone communications and 6 declined to participate in the physical screening. 45 consented to physical screening, from which 36 were eligible and enrolled in the study. The most common reasons for ineligibility via online screening were current foot orthoses use $(n=70,53 \%)$, previous lower limb injury or surgery $(n=29,22 \%)$ and other sources of anterior knee pain $(n=18,14 \%)$. The most common reason for exclusion after physical screening was the presence of other sources of anterior knee pain (Osgood Schlatter's Disease, $n=6$; patellar tendinopathy, $n=2$ ). In March 2020, recruitment was ceased at 36 participants due to COVID-19 restrictions.

Of the 36 participants randomised, 17 participants were allocated to receive contoured foot orthoses and 19 participants were allocated to flat insoles. 13 (76\%) participants from the contoured foot orthoses group and 12 (63\%) participants from the flat insole group attended appointments with their physiotherapist and were fitted

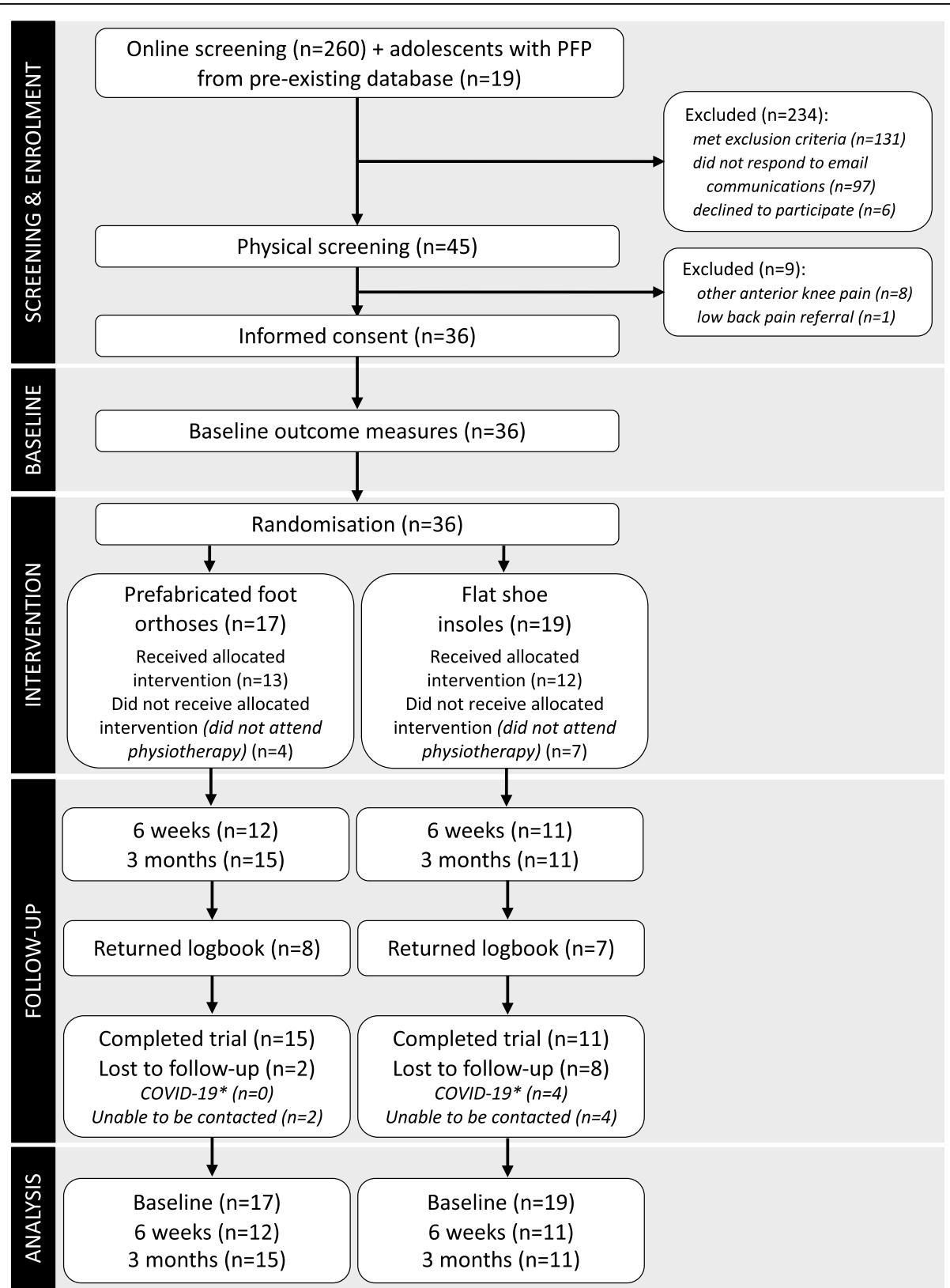

Fig. 1 Participant flow through the study. ${ }^{*}$ COVID-19 loss to follow-up refers to those whose participation in the trial was affected by COVID-19 restrictions 
with their allocated intervention. The mean number of appointments attended was 2.5 (SD 0.6) (contoured foot orthoses group 2.6 (0.7); flat insole group $2.4(0.5)$ ), and the mean number of inserts fitted was $2.6(0.8)$ (contoured foot orthoses group 2.7 (1); flat insole group 2.6 (0.7)). The remaining 11 participants did not attend physiotherapy appointments, and did not receive their allocated intervention. 3-month outcome measures were completed by $15(88 \%)$ participants from the contoured foot orthoses group and 11 (58\%) participants from the flat insole group ( $72 \%$ of total cohort). Participant characteristics are presented in Table 1.

\section{Feasibility results (primary outcomes)}

\section{Number of eligible volunteers, willingness to enrol, and} recruitment rate

$12.9 \%(36 / 279)$ of all volunteers (including those who responded to advertisements and those who were recruited from a pre-existing database) were eligible and willing to enrol in the study. Of the 45 volunteers who underwent physical screening, 36 (80\%) were eligible and provided informed consent to enrol in the study. Recruitment rate was 1.2 participants per week over 30 weeks.

\section{Adherence}

Fifteen of the 36 (42\%) participants completed and returned the paper logbooks; 8 out of 17 from the contoured foot orthoses group, and 7 out of 19 from the flat insole group (Fig. 1). From the logbooks, 7 out of 15 (47\%) participants met predefined minimum adherence with shoe insert wear ( $2 \mathrm{~h}$ per day, 5 days per week) for at least 7 out of 12 weeks; 5 participants from the contoured foot orthoses group, and 2 participants from the flat insole group. The logbook data showed that participants wore their allocated shoe inserts for a mean of 4.5 (SD 1.8) hours per day (contoured foot orthoses: 4.5 (1.5) hours per day; flat insoles: 4.4 (2.1) hours per day).

\section{Adverse events}

Only minor, transient adverse events were reported, predominantly in logbooks. In the contoured foot orthoses group, one participant reported one day of foot arch pain (week 3), and one participant reported experiencing blisters (weeks 1, 5 and 6) and one day of foot pain (week 2). In the flat insole group, two participants reported one day of blisters (week 1 and 2), and one participant reported foot pain on the first day of wear (week 1). One participant who received flat insoles contacted the investigators about the inserts rubbing in the second week of wear, causing minor discomfort and skin redness. This resolved in two days, and the participant was able to continue wearing the inserts. .

\section{Success of blinding}

Figure 2 presents data from the Credibility and Expectancy Questionnaire. There were no notable differences in median scores between groups at baseline or after being fitted with their allocated shoe inserts.

\section{Drop-out rate}

Dropout rate was $28 \%(10 / 36)$ at 3 months.

Table 1 Baseline characteristics of participants. Values are mean (SD) unless otherwise indicated

\begin{tabular}{|c|c|c|c|}
\hline Characteristics & Contoured foot orthoses $(n=17)$ & Flat shoe insoles $(n=19)$ & Total $(N=36)$ \\
\hline Age (years), median [IQR] & $14[5]$ & $17[3]$ & $15[4]$ \\
\hline Number (\%) of females & $12(71)$ & $15(79)$ & $27(75)$ \\
\hline Height (cm) & $165(9)$ & $169(12)$ & $167(10)$ \\
\hline Body mass (kg) & $58.5(12.1)$ & $60.8(14.4)$ & $59.7(13.3)$ \\
\hline Number (\%) with bilateral knee pain & $11(65)$ & $10(53)$ & $21(58)$ \\
\hline Duration of knee pain months, median [IQR] & $9[38]$ & 24 [18] & $18.5[29.5]$ \\
\hline Average pain severity ${ }^{\wedge}$, median [IQR] & $50[24]$ & $50[40]$ & 50 [34] \\
\hline Worst pain severity^, median [IQR] & $70[32]$ & $70[37]$ & $70[21]$ \\
\hline \multicolumn{4}{|l|}{ Most painful activity in the last week, count (\%): } \\
\hline squatting & $5(29)$ & $6(32)$ & $11(30.5)$ \\
\hline running & $6(35)$ & $5(26)$ & $11(30.5)$ \\
\hline walking upstairs & $4(24)$ & $4(21)$ & $8(22)$ \\
\hline walking downstairs & $2(12)$ & $1(5)$ & $3(8)$ \\
\hline walking & $0(0)$ & $2(11)$ & $2(6)$ \\
\hline jumping & $0(0)$ & $1(5)$ & 1 (3) \\
\hline
\end{tabular}

${ }^{\wedge}$ Measured on a $100 \mathrm{~mm}$ visual analogue scale $(0=$ no pain, $100 \mathrm{~mm}=$ worst pain imaginable), during their nominated most aggravating activity in the past week IQR interquartile range 


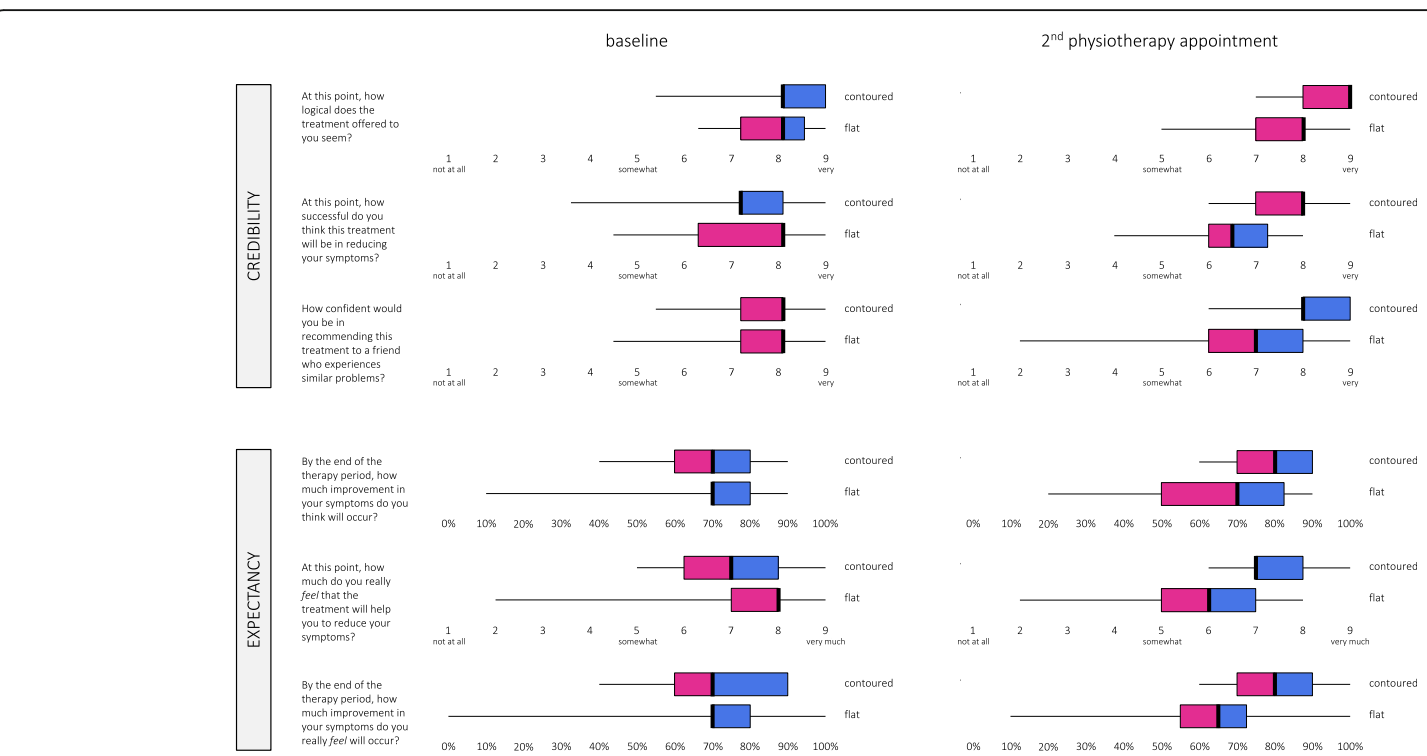

Fig. 2 Credibility and Expectancy Questionnaire data at baseline and 2nd physiotherapy appointment. Left whisker: quartile 1; pink box: quartile 2; black line: median; blue box: quartile 3; right whisker: quartile 4

Table 2 Secondary outcome measures at baseline, 6 weeks and 3 months (mean (SD) unless otherwise stated)

\begin{tabular}{|c|c|c|c|c|c|c|}
\hline & \multicolumn{3}{|c|}{ Contoured foot orthoses $(n=17)$} & \multicolumn{3}{|c|}{ Flat shoe insoles $(n=19)$} \\
\hline & Baseline & 6 weeks $^{\mathrm{a}}$ & 3 months $^{b}$ & Baseline & 6 weeks $^{c}$ & 3 months $^{d}$ \\
\hline Average pain severity [0-100 mm] & $47.7(16.1)$ & $46.1(21.7)$ & $38.1(21.2)$ & $47.5(23.2)$ & $40.6(23.5)$ & $33.4(20.7)$ \\
\hline Worst pain severity [0-100 mm] & $63.8(16.7)$ & $60(23)$ & $47.9(23.8)$ & $57.9(24.4)$ & $51.8(28.8)$ & $42.7(24.1)$ \\
\hline \multicolumn{7}{|l|}{ GROC, number (\%) } \\
\hline completely recovered & - & $0(0)$ & $1(6.5)$ & - & $0(0)$ & $0(0)$ \\
\hline strongly recovered & - & $4(33)$ & $8(53.5)$ & - & $3(27.3)$ & $4(36)$ \\
\hline slightly recovered & - & $5(42)$ & $4(27)$ & - & $3(27.3)$ & $5(46)$ \\
\hline same & - & $2(17)$ & $1(6.5)$ & - & $3(27.3)$ & $2(18)$ \\
\hline slightly worse & - & $0(0)$ & $0(0)$ & - & $1(9)$ & $0(0)$ \\
\hline much worse & - & $1(8)$ & $1(6.5)$ & - & $1(9)$ & $0(0)$ \\
\hline $\begin{array}{l}\text { Number (\%) responding 'yes' to Patient } \\
\text { Acceptable Symptom State }\end{array}$ & $12(71)$ & $9(75)$ & $13(87)$ & $13(68)$ & $7(64)$ & $8(73)$ \\
\hline \multicolumn{7}{|l|}{ KOOS-Child [100-0] } \\
\hline Pain & $63.4(10.6)$ & $66.7(14.4)$ & $81.3(12.3)$ & $63.5(16.8)$ & $68.2(19.1)$ & 79 (13.7) \\
\hline Symptoms & $77.5(9.9)$ & $77.1(13.4)$ & $88.5(10.1)$ & $75.4(13.2)$ & $77.6(12.7)$ & $84.7(12.1)$ \\
\hline$A D L$ & $82.2(9.5)$ & $86.4(10.6)$ & $94(6.5)$ & $79.1(14.5)$ & $87.2(9.7)$ & $93.8(7)$ \\
\hline Sport and play & $62.4(16.5)$ & $69.3(23.4)$ & $81.6(17.4)$ & $62.2(20.3)$ & $72.1(25.4)$ & $87.3(16.3)$ \\
\hline QoL & $57.1(17.1)$ & $61.1(20.6)$ & $78(17.8)$ & $52.9(18.5)$ & $64(20)$ & $75.4(18.5)$ \\
\hline KOOS-PF [100-0] & $60.6(15.8)$ & $65.1(23)$ & $82.6(13.7)$ & $58(16.9)$ & $70.9(17.4)$ & $80.4(19.6)$ \\
\hline Anterior Knee Pain Scale [100-0] & $74.7(10.7)$ & $75.5(13.6)$ & $85.5(10.2)$ & $72.6(10.8)$ & $79.4(13.3)$ & $87.5(9.8)$ \\
\hline EQ-5D-5L index value [1-0] & $0.72(0.1)$ & $0.68(0.28)$ & $0.83(0.13)$ & $0.71(0.19)$ & $0.76(0.13)$ & $0.85(0.12)$ \\
\hline EQ-5D-5L VAS [100-0] & $76.9(15.1)$ & $81.6(20.7)$ & $80.2(17.7)$ & $81.8(10.7)$ & 83 (12.6) & $88.4(7.6)$ \\
\hline
\end{tabular}

GROC global rating of change, KOOS Knee injury and Osteoarthritis Outcome Score, ADL activities of daily living, QoL quality of life, KOOS-PF KOOS patellofemoral subscale, VAS visual analogue scale

Square parentheses indicate possible score range, from best to worst possible score.

Number of observations: ${ }^{\mathrm{a}} n=12 ;{ }^{\mathrm{b}} n=15 ;{ }^{\mathrm{c}} n=11 ;{ }^{\mathrm{d}} n=11$. 


\section{Secondary outcomes}

Table 2 presents secondary patient-reported outcome measures at baseline, 6 weeks, and 3 months. Of the 15 participants who returned logbooks, 3 participants reported using co-interventions for their knee pain. One participant in the contoured foot orthoses group used paracetamol (1 occasion). In the flat insole group, one participant used paracetamol ( 3 occasions) and ice (1 occasion), and one participant used ice (15 occasions).

\section{Discussion}

Our findings indicate that a full-scale RCT of contoured foot orthoses versus flat insoles for adolescents with PFP would not be feasible with the protocol used in this study, based on our a priori criteria to inform feasibility. Although our observed recruitment rate (1.2 participants per week) exceeded the pre-specified criterion of 1 participant per week, parameters for minimum adherence with shoe insert wear and drop-out rate were not met. Of the 15 participants (42\%) who provided adherence data, only $7(47 \%)$ met minimum adherence of $2 \mathrm{~h}$ per day, 5 days per week for at least 7 out of 12 weeks. At 3 months, $28 \%$ of participants had dropped out (10/36), of which 4 were related to the COVID-19 pandemic. There were only minor, transient adverses event reported, and minimal co-intervention use. Participants perceived both interventions to be credible, and results of the Credibility and Expectancy Questionnaire demonstrated success of blinding. The method of patient-reported outcome collection was feasible and acceptable in this group.

Our recruitment strategy involved recruiting adolescent volunteers from the community via advertisements, as well as contacting adolescents with PFP from a preexisting database. Combined, this strategy led to a recruitment rate of 1.2 participants per week over 30 weeks. However, we acknowledge that a future full-scale RCT may not have access to such a database, and thus is likely to rely solely on recruitment from the community. If the 6 participants who were recruited from the preexisting database are not considered, our recruitment rate for the remaining 30 participants was 1 participant per week. This also meets our a priori criterion [17]. A full-scale trial in this population may need to run across multiple sites or use a broader recruitment strategy (e.g., through schools) to ensure efficient recruitment of the target sample size. Of the advertising methods used, social media was most successful, accounting for $90 \%$ of participants recruited from advertising (27/30). Future studies recruiting adolescents with PFP should consider using social media as their primary advertising method.

Our observed percentage of total volunteers entering the study (12.9\%) is in line with previous PFP RCTs. Rathleff et al. [13] reported a $16.7 \%$ inclusion rate of adolescents with knee pain at screening, who were recruited through schools. Collins et al. [11] included $11.7 \%$ of adult volunteers with PFP, who were recruited through community advertising. The most common reasons for excluding volunteers were that they were already wearing foot orthoses $(n=70)$, had sustained a previous lower limb injury $(n=29)$, or had anterior knee pain from another source (e.g. patellar tendinopathy, Osgood-Schlatter disease) $(n=18)$. Interestingly, 97 out of 260 (37\%) adolescents who responded to advertisements and completed the initial online screening form did not respond to follow-up email or telephone communications. Therefore, it is not possible to know how many of these volunteers were potentially eligible for physical screening. Our observed rate of non-response is substantially higher than the proportion of adults with PFP who did not respond to follow-up communications after volunteering for a previous study $(182 / 1530,11.9 \%)$ [11]. Although the reasons for this are unclear, adolescents are likely to have different considerations and preferences for communicating or engaging with researchers and barriers to participation, which need to be explored further.

We evaluated two aspects of adherence - logbook completion and time wearing the allocated inserts. $42 \%$ (15/36) of participants returned their logbooks, suggesting that data from the logbooks may not be representative of the whole group. Because the logbooks were the primary source of data relating to insert wear time, this also suggests that our insert adherence data may not be representative. $63 \%(5 / 8)$ of participants in the contoured foot orthoses group reported wear time that met our predefined criteria for minimum adherence for more than half of the 3-month study period, compared to $29 \%$ $(2 / 7)$ of participants from the flat insole group, although average wear time was similar for both groups ( 4.5 hours per day, or 32 hours per week). This is lower than a previous study in older adults with patellofemoral osteoarthritis (mean age 60 years), where logbooks were completed by $69 \%$ of participants and average insert wear time was 37 hours per week [35]. Our findings are likely to overestimate insert wear, as participants who completed and returned their logbooks may be more likely to be adherent with other aspects of the study, such as wearing their allocated insert. Further research is needed to determine the most effective methods of facilitating and monitoring adherence in this population. For example, app- or web-based methods may be more acceptable to adolescents with PFP due to the high use of electronic devices in this demographic [36]. Notwithstanding this, the low adherence rates that we observed may indicate that shoe inserts alone are not a viable intervention in this population. This requires further exploration to determine the barriers and facilitators to wearing shoe inserts, as well as footwear preferences of adolescents with PFP. For example, logbook data suggests that our participants spent a substantial amount of 
time wearing flip-flops or similar footwear, which coincided with days of low shoe insert wear time. Alternatively, there may be other factors that affected adherence with wearing the shoe inserts, such as the COVID-19 pandemic. Participants were in lockdown (confined to their homes) during the study period and were undertaking home schooling. This likely resulted in participants substantially reducing their shoe wear time, compared to normal school attendance and sport participation.

We set an a priori drop-out rate of $\leq 20 \%$ to inform feasibility [17]. Overall, $28 \%(10 / 36)$ of participants were lost to follow up at 3 months. 31\% (11/36) of participants did not attend any physiotherapy appointments, and therefore did not receive their allocated intervention. It is important to explore the reasons why these adolescents dropped out or did not attend physiotherapy. One consideration is the impact of the COVID-19 pandemic. Four participants were due to schedule their initial physiotherapy appointments when COVID-19 restrictions were coming into effect in Queensland. If these participants are not considered, then the total loss to follow-up is $17 \%(6 / 36)$, which is within our prespecified feasibility criterion, and the number of participants not attending any physiotherapy appointments is 7 (19\%). However, it is likely that there were other barriers to our participants attending physiotherapy appointments, such as relying on parents for transport and the geographic location of the clinics involved in our trial. These need to be addressed if a full-scale RCT is to be feasible.

There were minimal differences between groups on the Credibility and Expectancy Questionnaire. At baseline, prior to randomization, median scores for the two groups were identical or within 1 response category. After receiving the allocated intervention, the group who received flat insoles demonstrated lower scores on all items of the Credibility and Expectancy Questionnaire, although median scores for the two groups were within 1.5 categories. The similarity in scores between groups after receiving the intervention, and between baseline and follow up for item 6 (By the end of your therapy, how much improvement in your symptoms do you really feel will occur?) indicates success of blinding. No other studies have assessed credibility and expectancy outcomes in adolescents with PFP. However, success of blinding was demonstrated in a study investigating lower limb injury risk in military recruits randomized to receive contoured prefabricated foot orthoses or flat insoles [37]. This suggests that flat insoles are an appropriate comparator for foot orthoses, including for adolescents with PFP.

Our findings also indicate that adolescents with PFP have minimal adverse events associated with wearing shoe inserts. Six participants reported rubbing, blisters and foot pain associated with both types of shoe inserts, most often in the first 2 weeks of wear. However, these were minor, typically resolved in 1-2 days, and did not preclude ongoing insert wear. Similar to previous studies involving shoe inserts for knee pain [11, 15, 38], our findings indicate that contoured foot orthoses, as well as flat shoe inserts, are tolerated well by adolescents with PFP.

There are two key limitations of this study. Firstly, there was a higher drop-out rate observed in the flat insole group at 3 months ( $42 \%$ in flat insole vs. $12 \%$ in foot orthoses group). Half of the participants lost to followup in the flat insole group were due to the COVID-19 pandemic $(4 / 19,21 \%)$, prior to receiving their allocated inserts. We observed similar outcomes on the Credibility and Expectancy Questionnaire after participants had received their allocated shoe inserts, suggesting that there may be other factors affecting retention in this group. Secondly, only $42 \%$ of participants returned the logbook, resulting in incomplete data for adherence with the shoe inserts, use of co-interventions and pain relief, activities undertaken, and adverse reactions. Thus, it is not clear whether these data are representative of the entire cohort.

\section{Conclusion}

Based on a priori criteria for feasibility, our findings indicate that a full-scale RCT comparing contoured foot orthoses to flat insoles in adolescents with PFP would not be feasible using the current protocol, due to low rates of retention and adherence to the interventions. We recommend that feasibility issues are addressed prior to conducting a full-scale RCT, with protocol modifications to facilitate participant retention, logbook completion, and shoe insert wear.

\section{Abbreviations \\ CONSORT: Consolidated Standards of Reporting Trials; EQ-5D: EuroQOL-5D; EVA: Ethylene-vinyl acetate; GROC: Global Rating of Change; HAPPi Kneecaps!: sHoe inserts for Adolescents with Patellofemoral Paln; KOOS- Child: Knee injury and Osteoarthritis Outcome Score Child Version; KOOS- PF: Knee injury and Osteoarthritis Outcome Score patellofemoral subscale; PASS: Patient Acceptable Symptom State; PFP: Patellofemoral pain; \\ RCT: Randomised controlled trial; REDCap: Research Electronic Data Capture; SPIRIT: Standard Protocol Items: Recommendations for Interventional Trials}

\section{Acknowledgements}

We acknowledge Vasyli Medical for providing the foot orthoses and flat insoles used in this trial, as well as the contribution of study physiotherapists and practice staff.

\section{Authors' contributions}

NJC, KMC, SJK, MVM, AJS, BV, HBM and KT conceived the study. NJC, KMC, SJK and MVM obtained funding for the trial. All authors designed the trial protocol. ICO recruited participants and collected data, with assistance from KTO. ICO, NJC and AJS conducted data analyses, and all authors contributed to interpretation of findings. ICO and NJC drafted the manuscript, with input from KMC, SJK, MVM, BV, MMFS, HBM, AJS, KT, KTO and NCC. All authors have read and approved the final manuscript. 


\section{Funding}

This study was supported by an Arthritis Australia Project Grant (Molly Saint Grant), and a New Staff Start-up Grant from the School of Health and Rehabilitation Sciences, The University of Queensland. Isobel O'Sullivan is supported by a UQ Graduate School Scholarship. The study was designed independently of the funding bodies. Steven Kamper is supported by a National Health and Medical Research Council of Australia (NHMRC) Emerging Leadership Fellowship (ID: 1194769). Melinda Franettovich Smith was supported by a University of Queensland Development Fellowship (ID: UQFEL 1832429). Hylton Menz is supported by a National Health and Medical Research Council of Australia Senior Research Fellowship (ID: 1135995).

\section{Availability of data and materials}

The datasets used and/or analysed during the current study are available from the corresponding author on reasonable request.

\section{Declarations}

\section{Ethics approval and consent to participate}

Ethics approval for this study was granted by The University of Queensland Human Research Ethics Committee (approval number 2018000159). All participants (and their parent/guardian if aged under 18 years) provided written informed consent prior to participation.

\section{Consent for publication}

Not applicable.

\section{Competing interests}

BV is a voluntary (non-compensated) member by invitation on the Vasyli Think Tank. All other authors declare that they have no competing interests.

\section{Author details}

'School of Health and Rehabilitation Sciences: Physiotherapy, The University of Queensland, Brisbane, Australia. ${ }^{2}$ La Trobe Sport and Exercise Medicine Research Centre, School of Allied Health, Human Services and Sport, La Trobe University, Melbourne, Australia. ${ }^{3}$ Faculty of Medicine and Health, The University of Sydney, Sydney, Australia. ${ }^{4}$ Nepean Blue Mountains Local Health District, Penrith, Australia. ${ }^{5}$ Department of General Practice, Erasmus MC Medical University Center, Rotterdam, The Netherlands. ${ }^{6}$ School of Allied Health, Human Services and Sport, La Trobe University, Melbourne, Australia. ${ }^{7}$ School of Allied Health, Curtin University, Perth, Australia. ${ }^{8}$ School of Biomedical Sciences, The University of Queensland, Brisbane, Australia. ${ }^{9}$ Surgical Treatment and Rehabilitation Services, Metro North Hospital and Health Service, Herston, Australia. ${ }^{10}$ Faculty of Health and Behavioural Sciences, The University of Queensland, Brisbane, Australia.

\section{Received: 29 September 2021 Accepted: 10 November 2021} Published online: 10 December 2021

\section{References}

1. Smith BE, Selfe J, Thacker D, Hendrick P, Bateman M, Moffatt F, et al. Incidence and prevalence of patellofemoral pain: A systematic review and meta-analysis. PLoS One. 2018;13(1):e0190892-e.

2. Rathleff MS, Roos EM, Olesen JL, Rasmussen S, Arendt-Nielsen L. Lower mechanical pressure pain thresholds in female adolescents with patellofemoral pain syndrome. J Orthop Sports Phys Ther. 2013;43(6):41421. https://doi.org/10.2519/jospt.2013.4383.

3. Rathleff MS, Holden S, Straszek CL, Olesen JL, Jensen MB, Roos EM. Five-year prognosis and impact of adolescent knee pain: a prospective populationbased cohort study of 504 adolescents in Denmark. BMJ Open. 2019;9(5).

4. Rathleff CR, Baird WN, Olesen JL, Roos EM, Rasmussen S, Rathleff MS. Hip and knee strength is not affected in 12-16 year old adolescents with patellofemoral pain - A cross-sectional population-based study. PLoS One. 2013;8(11).

5. Molgaard C, Rathleff MS, Simonsen O. Patellofemoral pain syndrome and its association with hip, ankle, and foot function in 16- to 18-year-old high school students: a single-blind case-control study. J Am Podiatr Med Assoc. 2011;101(3):215-22. https://doi.org/10.7547/1010215.

6. Selhorst M, Fernandez-Fernandez A, Schmitt L, Hoehn J. Adolescent psychological beliefs, but not parent beliefs, associated with pain and function in adolescents with patellofemoral pain. Phys Ther Sport. 2020;45: 155-60. https://doi.org/10.1016/j.ptsp.2020.07.003.

7. Kastelein M, Luijsterburg PAJ, Heintjes EM, van Middelkoop M, Verhaar JAN, Koes BW, et al. The 6-year trajectory of non-traumatic knee symptoms (including patellofemoral pain) in adolescents and young adults in general practice: a study of clinical predictors. Br J Sports Med. 2015;49(6):400-5. https://doi.org/10.1136/bjsports-2014-093557.

8. Collins NJ, Barton CJ, van Middelkoop M, Callaghan MJ, Rathleff MS, Vicenzino BT, et al. 2018 consensus statement on exercise therapy and physical interventions (orthoses, taping and manual therapy) to treat patellofemoral pain: recommendations from the 5th international patellofemoral pain research retreat, Gold Coast, Australia, 2017. Br J Sports Med. 2018;52(18):1170-8. https://doi.org/10.1136/bjsports-2018-099397.

9. Rathleff M, Vicenzino B, Middelkoop M, Graven-Nielsen T, Linschoten R, Hölmich P, et al. Patellofemoral pain in adolescence and adulthood: same same, but different? Sports Med. 2015;45(11):1489-95. https://doi.org/10.1 007/s40279-015-0364-1.

10. Neal BS, Lack SD, Lankhorst NE, Raye A, Morrissey D, van Middelkoop M. Risk factors for patellofemoral pain: a systematic review and meta-analysis. Br J Sports Med. 2019;53(5):270-81. https://doi.org/10.1136/bjsports-2017098890.

11. Collins N, Crossley K, Beller E, Darnell R, McPoil T, Vicenzino B. Foot orthoses and physiotherapy in the treatment of patellofemoral pain syndrome: randomised clinical trial. BMJ. 2008;337(5):a1735. https://doi.org/10.1249/01. mss.0000321711.35702.2b.

12. Van Linschoten R, Van Middelkoop M, Berger MY, Heintjes EM, Verhaar JAN, Willemsen SP, et al. Supervised exercise therapy versus usual care for patellofemoral pain syndrome: an open label randomised controlled trial. BMJ (Online). 2009;339(7728):1010-3. https://doi.org/10.1136/bmj.b4074.

13. Rathleff MS, Roos EM, Olesen JL, Rasmussen S. Exercise during school hours when added to patient education improves outcome for 2 years in adolescent patellofemoral pain: a cluster randomised trial. Br J Sports Med. 2015;49(6):406-12. https://doi.org/10.1136/bjsports-2014-093929.

14. Eng JJ, Pierrynowski MR. Evaluation of soft foot orthotics in the treatment of patellofemoral pain syndrome. Phys Ther. 1993;73(2):62-8; discussion 8-70. https://doi.org/10.1093/ptj/73.2.62.

15. Mills K, Blanch P, Dev P, Martin M, Vicenzino B. A randomised control trial of short term efficacy of in-shoe foot orthoses compared with a wait and see policy for anterior knee pain and the role of foot mobility. Br J Sports Med. 2012;46(4):247-52. https://doi.org/10.1136/bjsports-2011-090204.

16. Collins NJ, Bierma-Zeinstra SM, Crossley KM, van Linschoten RL, Vicenzino $B$, van Middelkoop M. Prognostic factors for patellofemoral pain: a multicentre observational analysis. Br J Sports Med. 2013;47(4):227-33. https://doi.org/1 0.1136/bjsports-2012-091696.

17. O'Sullivan IC, Crossley KM, Kamper SJ, van Middelkoop M, Vicenzino B, Franettovich Smith MM, et al. HAPPi kneecaps! Protocol for a participantand assessor-blinded, randomised, parallel group feasibility trial of foot orthoses for adolescents with patellofemoral pain. J Foot Ankle Res. 2020; 13(1):50. https://doi.org/10.1186/s13047-020-00417-9.

18. Chan AW, Tetzlaff JM, Gotzsche PC, Altman DG, Mann H, Berlin JA, Dickersin K, Hrobjartsson A, Schulz KF, Parulekar WR, Krleza-Jeric K, Laupacis A, Moher D SPIRIT 2013 explanation and elaboration: guidance for protocols of clinical trials. BMJ. 2013;346:e7586, jan08 15, DOI: https://doi.org/10.1136/bmj.e7586.

19. Eldridge SM, Chan CL, Campbell MJ, Bond CM, Hopewell S, Thabane L, et al. CONSORT 2010 statement: extension to randomised pilot and feasibility trials. Pilot Feasibility Stud. 2016;2(1):64. https://doi.org/10.1186/s40814-0160105-8.

20. Aaron M. Orkin ea. guidelines for reporting trial protocols and completed trials modified due to the COVID-19 pandemic and other extenuating circumstances the CONSERVE 2021 statement. JAMA. 2021;326(3):257-65. https://doi.org/10.1001/jama.2021.9941.

21. Arain M, Campbell MJ, Cooper CL, Lancaster GA. What is a pilot or feasibility study? A review of current practice and editorial policy BMC Med Res Methodol. 2010;10(1):67. https://doi.org/10.1186/1471-2288-10-67.

22. Billingham SA, Whitehead $A L$, Julious $S A$. An audit of sample sizes for pilot and feasibility trials being undertaken in the United Kingdom registered in the United Kingdom clinical research network database. BMC Med Res Methodol. 2013;13(1):104. https://doi.org/10.1186/1471-2288-13-104.

23. Collins NJ, Tan JM, Menz HB, Russell TG, Smith AJ, Vicenzino B, et al. The FOOTPATH study: protocol for a multicentre, participant- and assessor-blind, parallel group randomised clinical trial of foot orthoses for patellofemoral 
osteoarthritis. BMJ Open. 2019;9(4):e025315. https://doi.org/10.1136/ bmjopen-2018-025315.

24. Borkovec TD, Nau SD. Credibility of analogue therapy rationales. J Behav Ther Exp Psychiatry. 1972;3(4):257-60. https://doi.org/10.1016/0005-7916(72 190045-6.

25. Devilly GJ, Borkovec TD. Psychometric properties of the credibility/ expectancy questionnaire. J Behav Ther Exp Psychiatry. 2000;31 (2):73-86. https://doi.org/10.1016/50005-7916(00)00012-4.

26. Clarke G, McGlinchey EL, Hein K, Gullion CM, Dickerson JF, Leo MC, et al. Cognitive-behavioral treatment of insomnia and depression in adolescents: a pilot randomized trial. Behav Res Ther. 2015;69:111-8. https://doi.org/10.1 016/j.brat.2015.04.009.

27. Turner CM, Mataix-Cols D, Lovell K, Krebs G, Lang K, Byford S, et al. Telephone cognitive-behavioral therapy for adolescents with obsessivecompulsive disorder: a randomized controlled non-inferiority trial. J Am Acad Child Adolesc Psychiatry. 2014;53(12):1298-307 e2. https://doi.org/10.1 016/j.jaac.2014.09.012.

28. Crossley KM, Bennell KL, Cowan SM, Green S. Analysis of outcome measures for persons with patellofemoral pain: which are reliable and valid? Arch Phys Med Rehabil. 2004;85(5):815-22. https:/doi.org/10.1016/S0003-9993(03)00613-0.

29. Ortqvist M, Roos EM, Brostrom EW, Janarv PM, Iversen MD. Development of the knee injury and osteoarthritis outcome score for children (KOOS-child): comprehensibility and content validity. Acta Orthop. 2012;83(6):666-73. https://doi.org/10.3109/17453674.2012.747921.

30. Crossley KM, Macri EM, Cowan SM, Collins NJ, Roos EM. The patellofemoral pain and osteoarthritis subscale of the KOOS (KOOS-PF): development and validation using the COSMIN checklist. Br J Sports Med. 2018;52(17):1130-6. https://doi.org/10.1136/bjsports-2016-096776.

31. Kvien TK, Heiberg T, Hagen KB. Minimal clinically important improvement/ difference (MCII/MCID) and patient acceptable symptom state (PASS): what do these concepts mean? Ann Rheum Dis. 2007;66(Suppl 3):iii40-1.

32. Kujala UM, Jaakkola LH, Koskinen SK, Taimela S, Hurme M, Nelimarkka O. Scoring of patellofemoral disorders. Arthroscopy. 1993;9(2):159-63. https:// doi.org/10.1016/50749-8063(05)80366-4.

33. Rabin R, de Charro F. EQ-5D: a measure of health status from the EuroQol group. Ann Med. 2001;33(5):337-43. https://doi.org/10.3109/078538901 09002087.

34. Sim J. Should treatment effects be estimated in pilot and feasibility studies? Pilot and Feasibility Studies. 2019;5(1):107. https://doi.org/10.1186/s40814-01 9-0493-7.

35. Tan JM, Menz HB, Crossley KM, Munteanu SE, Hart HF, Middleton KJ, et al. The efficacy of foot orthoses in individuals with patellofemoral osteoarthritis: a randomised feasibility trial. Pilot Feasibility Stud. 2019;5(1):90. https://doi.org/10.1186/s40814-019-0469-7.

36. Government A. Kids and mobiles: how Australian children are using mobile phones. Australian Communications and Media Authority. 2021.

37. Bonanno DR, Murley GS, Munteanu SE, Landorf KB, Menz HB. Effectiveness of foot orthoses for the prevention of lower limb overuse injuries in naval recruits: a randomised controlled trial. Br J Sports Med. 2018;52(5):298-302. https://doi.org/10.1136/bjsports-2017-098273.

38. Wyndow N, Collins NJ, Vicenzino B, Tucker K, Crossley KM. Foot and ankle characteristics and dynamic knee valgus in individuals with patellofemoral osteoarthritis. J Foot Ankle Res. 2018;11(1):65. https://doi.org/10.1186/s1304 7-018-0310-1.

\section{Publisher's Note}

Springer Nature remains neutral with regard to jurisdictional claims in published maps and institutional affiliations.

Ready to submit your research? Choose BMC and benefit from:

- fast, convenient online submission

- thorough peer review by experienced researchers in your field

- rapid publication on acceptance

- support for research data, including large and complex data types

- gold Open Access which fosters wider collaboration and increased citations

- maximum visibility for your research: over $100 \mathrm{M}$ website views per year

At BMC, research is always in progress.

Learn more biomedcentral.com/submissions 